EPiC Series in Computing
Volume 58, 2019, Pages 265-270
Proceedings of 34th International Confer-
ence on Computers and Their Applications

\title{
Parking Lot Occupancy Tracking Through Image Processing
}

\author{
Marcos Lopez ${ }^{1, *}$, Terry Griffin ${ }^{1, \dagger}$, Kevin Ellis ${ }^{1, \hbar}$, \\ Anthony Enem ${ }^{1, \S}$ and Christopher Duhan ${ }^{1, * *}$ \\ ${ }^{1}$ Midwestern State University \\ marcos.lopez@msutexas.edu, terry.griffin@msutexas.edu
}

\begin{abstract}
In this paper we approach the problem of searching for available parking in busy lots. While research has been done to allow users to view where empty parking spaces are located, it often involves expensive methods that are difficult to maintain. We attempt to address these restrictions. We also develop a platform to disperse the information to the target users.
\end{abstract}

\section{Introduction}

In the commute to a school or office campus, a driver often must spend time circling a parking lot in the hopes of a free space. This increases the traffic in busy locations, such as densely populated neighborhoods or universities. In this paper we detail our attempts decrease the search times of drivers by allowing them to view where available parking spaces are located. Our system processes live images from a web connected camera and determines if a vehicle is currently stationed in each individual space of a parking lot. The results of this program are then relayed to the user in a multiplatform mobile application. Our goal is to produce a system that could constantly and accurately monitor a series of parking lots to determine the availability of parking spaces on a university campus.

This research project was the result of various students at Midwestern State University. The genesis of our project began after students complained about the amount of time it took them to find a suitable parking location on campus. In the Fall of 2017, two students agreed to take on the challenge of identifying available parking using image processing. After a literature review, the group saw that using digital image processing was an established problem with various approaches.

\footnotetext{
${ }^{*}$ Faculty Advisor: Mathematics Department. Corresponding author.

$\dagger$ Faculty Advisor: Computer Science Department. Oversaw and maintained databases and coding.

\$ Student: Worked with Anthony on designing algorithms and coding.

${ }^{\S}$ Student: Worked with Kevin on designing algorithms and coding.

** Student: Prepared first draft of paper and preformed literature review.
} 
A common approach to this problem is to use embedded sensors that require significant construction on the parking lot as well at noticeable cost. For example, in (Lee, Yoon, \& Ghosh, 2008), a wireless sensor network is used that involve motion sensors under the spaces. Park et al. (Park, Kim, Seo, Kim, \& Lee, 2008) use mounted ultrasonic sensors to create their images by implementing an echo function.

While there are many ways that hardware systems can count the number of cars in a lot, they rarely offer information on a space by space level and are expensive to implement. Researchers have tried to improve upon the design of hardware systems by incorporating software solutions. Al-Kharusi and Al-Bahadly presented a combination solution in (Al-Kharusi \& Al-Bahadly, 2014) that requires a marking of each space with paint to be effective. Yusnita, Norbaya and Basharuddin present a method in (Yusnita, Norbaya, \& Basharuddin, 2012) that also uses a camera to recognize shapes that have been painted on the actual parking lot to determine if a space is available. A centralized control system is used in (USA Patent No. 6,266,609, 2001) that requires vehicles to have a navigation system. This paper will attempt to present an image classifier that only uses a mounted camera and software to make its classifications, instead of complicated sensors or the need for any interaction with the actual parking lot.

Wu and Zhang (Wu \& Zhang, 2006) approached this problem by using a multi-class support vector machine (SVM) in conjunction with a Markov random field. True also used an SVM and analysis of color histograms to classify parking spaces in (True, 2007). However, many researchers are looking for a system with easy implementation to their lots. For example, Magansal (Magansal, 2010) uses a counting scheme through image processing to answer this question. Color detection was also a common approach to lot classification. Hussin et al. (Hussin, Juhari, \& Wei, 2012) use color detection method, along with edge detection, to increase their accuracy.

Our method most closely aligns with the approach of Fabian in (Fabian, 2008). However, his approach considered shadows and attempted to eliminate them through image distortion. The Midwestern State research group also approached this problem with color detection. After the results were poor for certain types of vehicles, an edge detection module was added. In this paper, we will outline the components of our platform, and the modifications that have been made to them.

\section{Obtaining Parking Spaces}

While the goal of the research group is to provide live updates on parking availability at our local campus, initial findings were done at other locations. Unable to source a powered web camera that would be accessible locally, openly available web camera feeds were used to amass a database of parking lots. We identified three parking lots that provided a variety of weather, lighting, occupancy, age, and orientation.

A parking lot from the West Virginia University College of Engineering and Mineral Resources was selected to begin initial tests. Using the website Opentopia (Opentopia. Free Live Webcams. West Virginia Parking Lot., 2018), images were downloaded in ten-minute intervals over the course of October 2016 until February 2017 to later be used in the analysis of parking occupancy.

Since the camera remained stationary, each space was identified with the self-drawn boundary points that were recorded in a JSON file. The boundary points were selected as four corners of the empty space and were used to define a quadrilateral representative of the individual space. As a result, each space in the parking lot was able to be uniquely identified by its boundary points.

In addition to the usual labeled parking spaces, a vacant square of the parking lot was selected outside of normal spaces for comparison purposes. This space was specifically selected to be the uniform gray of the parking lot concrete. 
Once the parking lot had been entered into a database for labeling, a classifier was designed using color heuristics. For this classifier, all spaces (including the vacant square) were normalized in size and shape, so that they would each be formatted into a 40x40 pixel image. This was achieved by finding the smallest rectangle that contained all identifying boundary points of the space, filling all space outside of the unique quadrilateral with black pixels, and resizing this image to its appropriate size.

Using the OpenCV library in Python, each image was analyzed for color by recording the red, green, and blue (RGB) pixel values throughout each image. A histogram was then produced showcasing the frequency of each color value on the individual RGB channels. The hypothesis was that empty spaces would not a very diverse color palette compared to spaces occupied by colorful vehicles.

As can be seen in Figure 1 Histogram of gray vacant square. and Figure 2, there is a striking visual difference between the histograms of the preselected vacant space, and one occupied by a colorful vehicle.

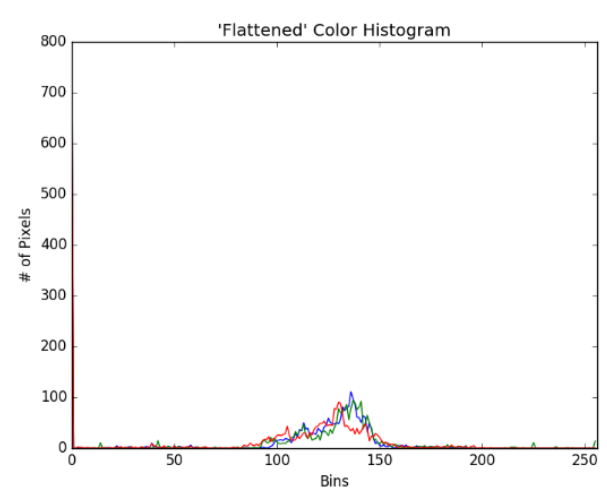

Figure 1 Histogram of gray vacant square.

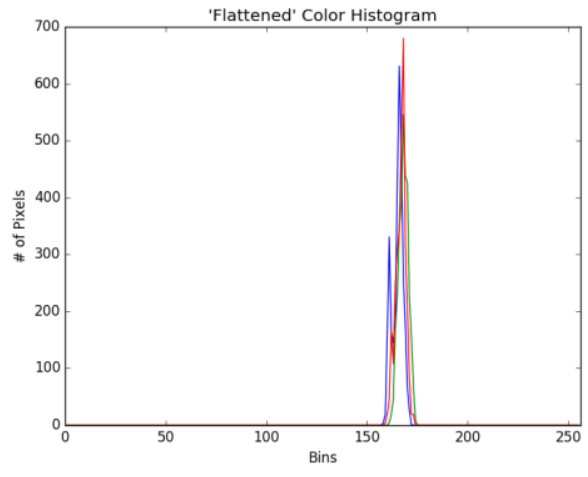

Figure 2 Histogram of occupied parking space.

The average color for each space was calculated on each channel to obtain an average color given in RGB format by summing up the pixel values for each RGB channel and dividing by total pixels in the image. This created a 3 -tuple that stored the average color of each channel as a vector.

A threshold, $\varepsilon$, was determined so that if the difference between the average color of the grey vacant space and the suspected parking space, Equation (1), was larger than $\varepsilon$, then the system would assume the space was occupied.

$$
\text { Difference }=\left|\left(\begin{array}{c}
B_{\text {avg }} \\
G_{\text {avg }} \\
R_{\text {avg }}
\end{array}\right)-\left(\begin{array}{c}
B_{\text {gray }} \\
G_{\text {gray }} \\
R_{\text {gray }}
\end{array}\right)\right|
$$

There were 10 parking lot images from the West Virginia campus that this algorithm was run on. This method proved good at identifying vehicles with dark coloring. However, lighter color models would give false negatives for occupancy. With an accuracy of approximately $50 \%$, depending on the concentration of light modeled vehicles, this method left much to be desired. A typical example of visualized output can be seen in Figure 3 Visualized output using color difference.. Green is suspected vacant, while red is suspected occupied. 


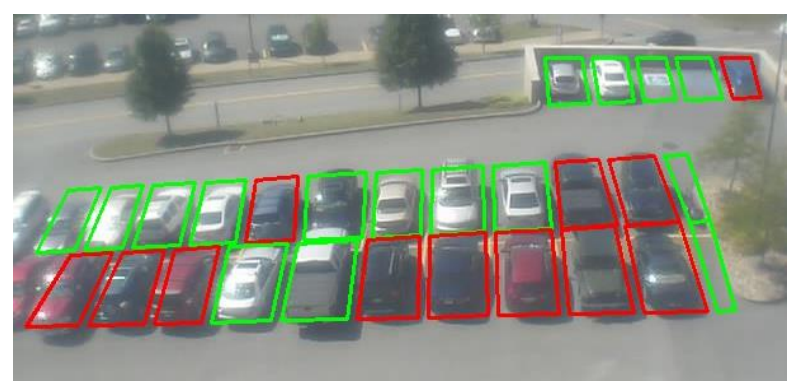

Figure 3 Visualized output using color difference.

This method had other drawbacks as well. One was the introduction of shadows would alter the average color of some spaces and not others. This gave an inconsistent reading on a lot. The amount of light throughout the day was also not constant, with the midday images having more glare and distorting the histograms. To combat this problem, the threshold value would also need to be moved throughout the day to adjust for the change in light levels from morning to evening. This prompted a second method using edge detection.

\section{Edge Detection}

For the edge detection method, an open source canny edge package from OpenCV was implemented. Our normalized photos of each space were converted into a black and white image highlighting the suspected edges in the original photo. If there was a high ratio of edge pixels to the overall pixel count of the image, it was deduced that a vehicle was in the space. A threshold value, $\varepsilon$, was again selected and could be modified to correspond with a particular parking lot. That is, if equation (2) was satisfied, the program would determine the space was occupied.

$$
\frac{\text { white pixels }}{\text { white pixels+black pixels }}>\epsilon
$$

The reader may notice the difference in white pixels between empty and occupied spaces in Figure . While there are edges detected in both images, it is clear the image on the right has far more white pixels, and the reader may be able to see characteristics of a vehicle.

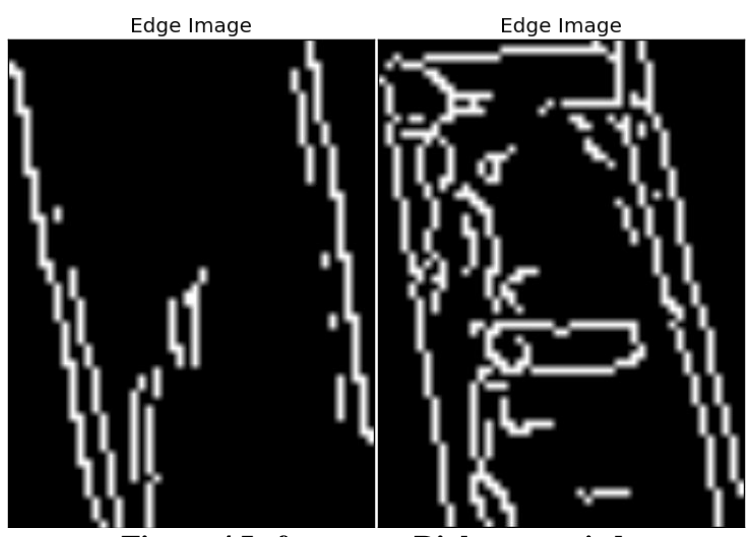

Figure 4 Left: empty. Right: occupied. 
This method had better results than using color heuristics on lighter colored vehicles, but still produced false negatives often. It was difficult to see edges in low lighting or dark colored vehicles. Edge detection also gave several false positives as a result. Figure is a typical visualized output of using the edge detection method. This was run on a limited number of parking lot images for the purposed of gaining knowledge in its effectiveness. Accuracy averaged at $62 \%$ for the 10 images tested. This average depended on the concentration of light vehicles to dark vehicles present in the lot.

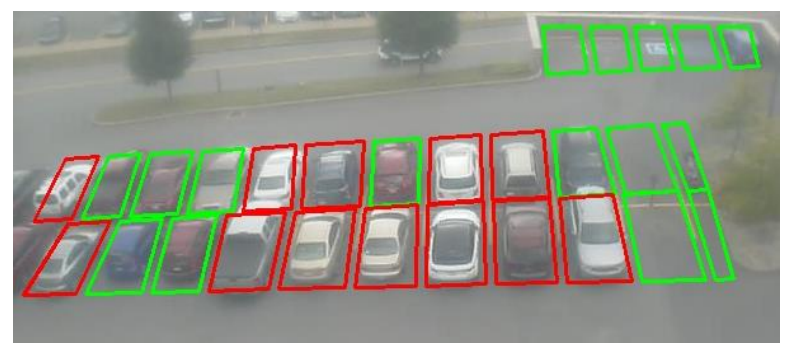

Figure 5 Visualized output using edge detection.

Another setback that was noticed was that if the parking lot being analyzed was poorly maintained and had many cracks, the cracks would be detected as edges, and the space labeled as occupied. However, on a smooth lot, the number of false positives for edge detection and color heuristics were both minimal.

To play into the strength of each method, both were used in parallel on a parking lot. This combined method labeled a parking space occupied if either the color heuristics or edge detection approach deemed the space occupied. Combining the two methods led had the true positives of the color method on darker cars, and the true positives of the edge detection for the lighter vehicles. The combined algorithm was then run on a larger variety of the West Virginia images. The new accuracy averaged $86 \%$, with a typical output found in Figure .

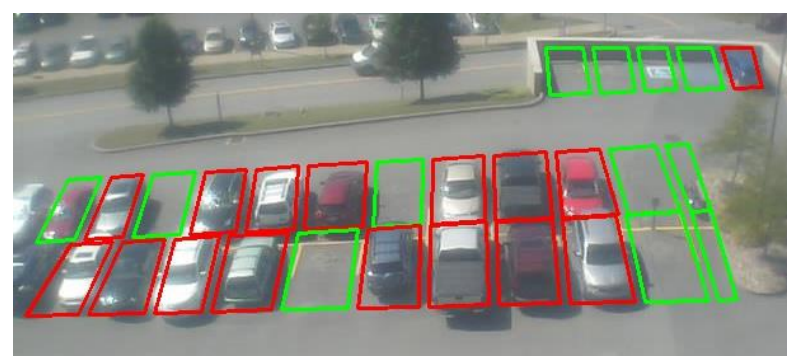

Figure 6 Visualized output using combined method.

\section{Conclusion and Future Work}

The combined method of parking lot classification has a low accuracy for the purposes of a commercial application but may prove useful for smaller-scale projects. Our aims were to have a method that would correctly predict a space's occupancy over $99 \%$ of the time.

The research group decided that machine learning may offer a new approach that would not have the same problems as the combined method. It was determined to pursue a machine learning approach and incorporate a convolutional neural network into our image classifier. 
With a machine learning approach, a new parking lot could be monitored almost immediately, without the necessity of finding appropriate threshold values and how they could best be adjusted throughout the day of changing light conditions.

Initial use of a neural network with a single hidden layer returned results of over $98 \%$ on two different lots based on a small testing database. The research group decided to pursue an increase in accuracy with a more robust neural network, and to build a large database of pre-classified parking spaces in which to train said neural network.

To achieve these goals, several smaller tasks have been undertaken by the research group. A parking lot classifier will be built to build up the necessary database to properly train a neural network. The neural network will be designed and implemented into a mobile application interface for students of Midwestern State University.

\section{Acknowledgements}

This Project has been supported by Midwestern State University under its Enhancing Undergraduate Research Endeavors \& Creative Activities (EURECA) program. The authors would like to thank the EURECA program and staff for helping the initialization of this project.

\section{References}

Al-Kharusi, H., \& Al-Bahadly, I. (2014). itelligent Parking Management System Based on Image Processing. World Journal of Engineering and Technology, 2, 55-67.

Fabian, T. (2008). An algorithm for parking lot occupation detetction. Computer Information Systems and Industrial Management Applications (pp. 165-170). IEEE.

Fastenrath, U. (2001). Parking Space Detection. USA Patent No. 6,266,609.

Hussin, R., Juhari, M., \& Wei, N. (2012). Digital image processing techniques for object detection from complex background image. Procedia Engineering, 41, 340-344.

Lee, S., Yoon, D., \& Ghosh, A. (2008). Intelligent parking lot application using wireless sensor networks. Collaborative Technologies and Systems (pp. 48-54). CTS2 008: IEEE.

Magansal, J. B. (2010). Counting available parking space using image processing. PhD Dissertation.

Opentopia. Free Live Webcams. West Virginia Parking Lot. (2018, November). Retrieved from Opentopia.com: http://www.opentopia.com/webcam/11480

Park, W.-J., Kim, B.-S., Seo, D.-E., Kim, D.-S., \& Lee, K.-H. L. (2008). Parking space detection using ultrasonic sensor in parking assistance system. Intelligent Vehicles Symposium (pp. 103-1044). 2008 IEEE: IEEE.

True, N. (2007). Vacant parking space detection in static images. University of California, San Diego, 17.

Wu, Q., \& Zhang, Y. (2006). Parking lots space detection. Machine Learning, Fall, 6.

Yusnita, R., Norbaya, F., \& Basharuddin, N. (2012). Intelligent Parking Space Detection System Based on Image Processing. International Journal of Innovation, Management and Technology, 3, 232. 